\title{
Comparative Experiments on Simulated Tornado Experience via Virtual Reality and Augmented Reality
}

\author{
Hiroyuki Mitsuhara* and Masami Shishibori*
}

(Received 26 June 2019 and accepted in revised form 5 February 2020)

\begin{abstract}
In Japan, a tornado is an infrequent natural disaster; however, tremendous winds can cause considerable damage. To prepare for such events, simulated tornado experience (STE) considering tremendous wind as a major hazard and incorporating evacuation plans must be provided. However, introduction of a large wind generator in STE is difficult. We developed simple VR and AR systems (VR-STE and AR-STE) that render STE by realistically simulating tornadoes and conducted comparative experiments. We set research questions that aimed to clarify how VR- and AR-STEs differ from STE, which expresses tornadoes with audiovisual effects and real, tremendous wind generated by a large wind generator. The survey variables were fear, self-efficacy, and learning motivation to cope with a tornado. The experimental results revealed that audiovisual effects can retain learners' fear of a tornado and influence learners' self-efficacy and learning motivation. Furthermore, VR-STE is more suitable than AR-STE in terms of controlling fear and system operation.
\end{abstract}

Keywords: augmented reality, fear, learning motivation, self-efficacy, tornado, virtual reality

\section{Introduction}

Natural disasters are threats that can cause human deaths. An insurance company reports ${ }^{(1)}$ that the number of occurrences of severe natural disasters is bound to increase worldwide. In Japan, frequently occurring severe natural disasters include earthquakes, typhoons, and floods. For instance, strong earthquakes with 4 or higher intensity (on the Japanese scale of 7) hit 78 times during $2018^{(2)}$. In such situations, the criticality of disaster management is indisputable. Disaster education is essential for protecting human lives since people may have to survive disasters on their own by taking appropriate decisions and adopting strategies using what they have learned to survive.

In recent years, natural disasters, and the resulting damage, have diversified due to climatic changes (e.g., global warming), environmental changes (e.g., deforestation), and social changes (e.g., depopulation). Disaster education should, therefore, be diversified. An approach to diversified disaster education is making efficient use of information and communication technology (ICT), i.e., ICT-based disaster education (ICTDE). A tsunami simulator that visualizes evacuees, along with damage expansion under various conditions, can be used for emergency planning ${ }^{(3)}$. A digital role-playing game can

\footnotetext{
*Graduate School of Technology, Industrial and Social Sciences, Tokushima University, Japan
}

provide engaging and effective learning of ethical decision-making during disaster response by giving players game-style missions in a virtual disaster world ${ }^{(4)}$. Furthermore, a digital shared map, where residents upload local information through fieldwork, can be used to protect the community from disasters ${ }^{(5)}$. By introducing virtual $(\mathrm{VR})^{(6)}$ and augmented realities $(\mathrm{AR})^{(7)}$, evacuation training can enhance simulated evacuation experience. Besides, ICTDE strongly promotes practiceoriented learning.

ICTDE should cover not only frequent but also infrequent, natural disasters because people who are not educated on infrequent natural disaster management may not survive if they encounter them. In Japan, a tornado is an infrequent natural disaster; the annual mean number and deaths from tornadoes during 2007-2015 was twenty-five and four, respectively ${ }^{(8)}$. Compared with frequent natural disasters, these numbers may not be remarkable; however, strong winds during tornadoes have caused considerable damages. In 2012, for instance, an F3 tornado (on Fujita scale of F0-F6) destroyed more than 200 houses in addition to one dead and thirty-seven injured persons ${ }^{(9)}$. Each time the media reports the occurrence of a tornado, people may worry about possible tornadoes; however, due to tornado infrequency, they believe that they would not encounter tornadoes.

We focus on how ICTDE can cover tornadoes. We developed simple VR and AR systems that provide a 
simulated tornado experience (STE) and then conducted comparative experiments using the systems. The survey items in the experiments include fear, self-efficacy, and learning motivation to cope with a tornado.

The remainder of this paper is organized as follows. Section 2 overviews the STE and presents some research questions in this direction. Section 3 explains the comparative experiments. Section 4 provides experimental results. Section 5 describes the considerations and answers the research questions. Finally, Section 6 summarizes the study and highlights future research.

\section{Simulated Tornado Experience}

Disaster education prompts people to imagine possible disasters, i.e., natural disasters that they may experience in their communities, and learn how to survive them. Despite knowing about past disasters, people have difficulty imagining such possibilities. In particular, most people in Japan have not experienced tornadoes and, thus, may not be able to imagine what it may be like. Hence, disaster education aims to supplement their imagination.

ICTDE is advantageous in terms of the immersiveness provided by three-dimensional computer graphics (3DCG) and head-mounted displays (HMDs), to mention a few.

\subsection{Fundamental Idea}

It is extremely difficult to foresee when and where a tornado will occur, even if there are presages such as a plunge in surrounding brightness, and an alert issued by, for example, the Japan Meteorological Agency (JMA). This shows that when a tornado occurs, people may not have enough time to make decisions for a successful evacuation. Thus, STE should incorporate learning how to evacuate.

\subsubsection{Steps}

People who are indoors when sighting a tornado only have to take obvious precautions, while those who are outdoors have to make decisions from various possibilities as they observe a tornado approaching. According to JMA's instructions, STE should focus on the immediate steps people outdoors should be taking: 1. A person sees a tornado approaching him or her.
2. The person enters the nearest stable building, i.e., makes a decision about the most suitable building for evacuation and rushes into the building; otherwise, he/she crouches in a place that is lower than its surroundings or behind a strong construction.

3. The person protects his or her head and neck while keeping away from windows.

\subsubsection{Evoking Fear}

Fear with its resulting anxiety is a major topic in disaster management. Rüstemli and Karanci revealed through interviews with earthquake victims (adults), that fear is significantly correlated with earthquake preparedness ${ }^{(10)}$. In disaster education, how to manage fear is a frequently discussed topic. Johnson et al. reviewed disaster education programs for children and reported that the programs did not show a consistent effect on the children's levels of fear ${ }^{(11)}$. Cvetković et al. investigated how young adults (university students) think about fear of disasters and concluded that better disaster education needs to shift from "threat" talk to "challenge" talk, as well as to contemporary survival strategies ${ }^{(12)}$. Nevertheless, disaster education has not reached a satisfactory conclusion on this matter.

We opine that fear is required to motivate people to learn evacuation skills. In other words, arousing fear in natural disasters leads to learning how to survive amidst the catastrophe. However, excessive fear can lower selfefficacy and learning motivation, as people may believe that surviving a tornado is impossible and, thus, there is no need to learn. We suggest that a moderate level of fear of tornadoes, i.e., not too much or too little, leads to increased self-efficacy and learning motivation. The moderate level of fear in STE can be expressed as follows (Figure 1):

- Not-too-much fear: A tornado occurs at a distant visi-

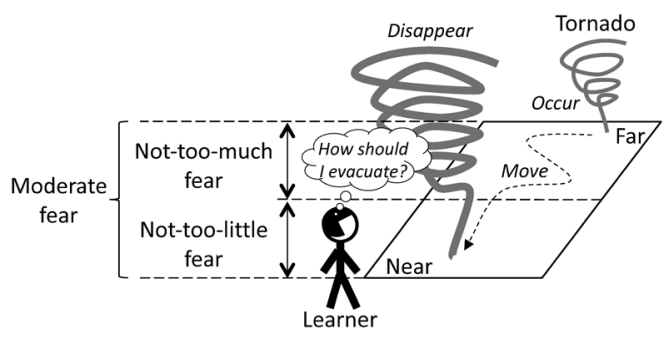

Figure 1. Moderate Fear in STE. 
ble location and approaches a learner. The learner can take Step 2, i.e., turn his or her thoughts to evacuation. As the tornado approaches, he/she recognizes an incremental need for taking Step 2.

- Not-too-little fear: The tornado comes extremely close to but does not engulf the learner. He/she must take Step 2 immediately. As a result, he/she can survive just in time.

\subsubsection{Expressing Tremendous Wind with Audiovisual Effects}

In order to evoke a sufficient level of fear of tornadoes, STE should include tremendous winds: as it becomes windy, the level of fear will increase. However, introducing a large wind generator in STE seems to be difficult. To highlight fear, ICTDE needs to provide STE in which a tornado, accompanied by tremendous wind, is realistically expressed with audiovisual effects, such as playing the sound of the wind roaring and displaying debris flying through the air. We refer to such STE as audiovisual STE.

A promising method for providing audiovisual STE is to use VR or AR; both can express various phenomena with their high expression of reality, immersion, and interactivity. In VR-based audiovisual STE, a 3DCG tornado is displayed in a virtual world, and learners move around and make various body movements in the virtual world using an intuitive device. In AR-based audiovisual STE, a 3DCG tornado is superimposed onto a real-time view captured by a camera-embedded device. Learners move and perform different actions in the real world. However, real-world spaces are required to satisfy Steps 2 and 3.

\subsection{Research Questions}

For VR- and AR-based audiovisual STEs, it is assumed that learners feel fear by seeing the tornado in Step 1. If this assumption is not satisfied, they may not be motivated to evacuate, i.e., they may not shift to Step 2.

We focused on audiovisual effects in Step 1 and asked the following research questions (RQs):

- (RQ1) Can audiovisual effects make learners feel fear of tornadoes?

- (RQ2) How can fear of tornadoes influence learners' self-efficacy and learning motivation?

- (RQ3) Which is more suitable: VR- or AR-based

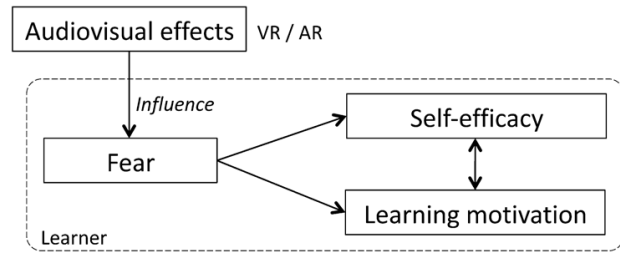

Figure 2. Audiovisual Effects, Fear, Self-efficacy, and Learning Motivation.

\section{audiovisual STE?}

In audiovisual STE, self-efficacy is associated with effective evacuation, i.e., ensuring the safety of the students facing the tornadoes. For example, students with high learning motivation may investigate possible tornado hazards or take note of stable buildings in their surroundings. Our model is simple and begins with audiovisual effects and has unidirectional effects ranging from fear to self-efficacy and learning motivation (Figure 2).

\subsection{Related Work}

There have been simulated disaster experience systems that considered fear or anxiety of disasters. Chittaro's research group developed a serious game system where users (players) can learn emergency evacuation in an attack situation while interactively moving around a three-dimensional virtual world. Their system aims to evoke fear by audiovisual effects, such as blood squirts, and simultaneously enhances self-efficacy by presenting appropriate evacuation strategies ${ }^{(13)}$. The same research group developed a VR-based serious game system that covers how to evacuate from a water crash-landed aircraft and adopts a similar method for fear $^{(14)}$. This system aroused more fear than the traditional evacuation instructions typically found on safety cards. Hirokane et al. developed an AR-based system that realistically expresses torrential rain with $3 \mathrm{DCG}$ animation and binaural recorded sound of rainfall. This system does not explicitly focus on fear but aims at improving risk prediction skills with realistic audiovisual effects ${ }^{(15)}$.

Other examples of built systems include: a first-person, 360-degree viewable VR content displays an F2 tornado that passes over a person staying in a house and destroys the house ${ }^{(16)}$; a VR-based serious game system 


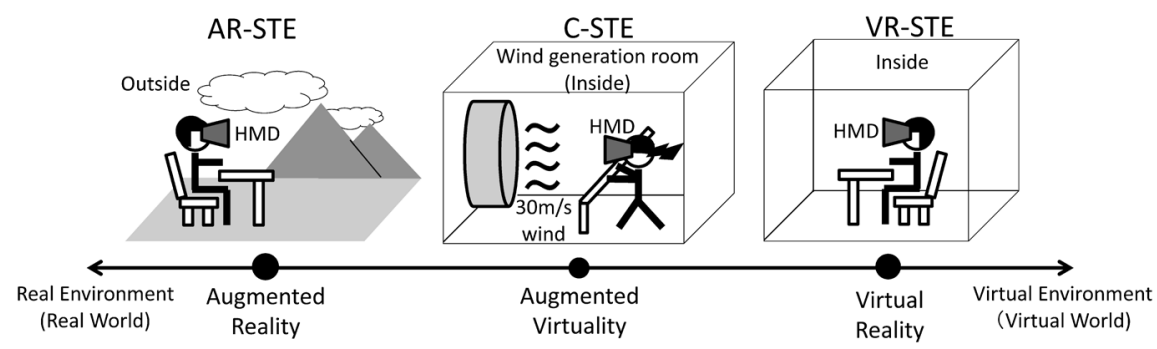

Figure 3. Overview of Experiments (Three STEs) Corresponding Reality-virtuality Continuum.

enables learners to perform tornado rescue missions as different characters, such as firefighters and medical responders, and heightens learners' motivation, engagement, and self-direction ${ }^{(17)}$; a smartphone-based AR system superimposes 3DCG of victims, fire, and tornadoes onto the real-time view to express rescue situations in mass casualty incidents ${ }^{(18)}$. VR and AR were used for STE, but there was little focus on evacuation. However, through tests, VR and AR-based STEs have been shown to not been extensive enough. In the virtual world, a VR-based STE can be a closed system not only expressing tornadoes but also surrounding landscapes under different conditions. For example, one can assert that a direct hit from a tornado will damage wooden houses. Hearing this may give rise to fear, but learners may lose that while immersed in an unknown virtual world, whereas with an AR-based STE, which combines a simulated tornado and the real world (e.g., their neighborhood), will communicate itself as if there were a tornado before their eyes. This kind of expression may inspire them to think of the possibility of encountering a tornado in their daily life. An AR-based STE, however, cannot express the influence of the tornado on a real landscape (e.g., houses destroyed). Thus, an essential difference between VR- and AR-based STEs is whether the damage of a tornado on the landscape can be expressed.

\section{Experiments}

We developed simple VR and AR systems that provide audiovisual STEs. We conducted comparative experiments using the systems at a public educational facility on disaster management. We adopted a questionnaire to survey the participants' fear, self-efficacy, and learning motivation.

\subsection{Design}

We formed two experimental groups: VR- and AR-based audiovisual STEs (VR-STE and AR-STE for short), and one control group. The control group used a VR system in a wind-generation room with a large wind generator and judging from the questionnaire results was observed to have the highest level of fear. The control group participants experienced $30 \mathrm{~m} / \mathrm{s}$ wind with audiovisual effects $-30 \mathrm{~m} / \mathrm{s}$ wind corresponds to a F0 tornado, i.e., a tornado of the smallest scale. We refer to VR-STE with real wind as a combination STE (C-STE). Based on a reality-virtual reality continuum ${ }^{(19)}$, C-STE can be placed at augmented virtuality that complements a virtual world with real wind. Figure 3 overviews the experiments that illustrate how VR-and AR-STEs differ from C-STE.

In C-STE, the wind generator was unable to spasmodically change the direction of the wind, and for safety reasons the participants always faced the wind. This situation may differ from an actual tornado drawing inward centripetally, together with turbulence in the wind. Most of the participants catching a veering wind from behind may be more realistic. However, in the experiments, we focused on wind power as a major fear element of tornadoes, not direction. This is because people may be more impressed by the strength of the wind. Therefore, in the experiments we did not explore directional fidelity.

\subsection{Developed Systems}

We developed the VR and AR systems using a game engine (Unity3D) and adopted a binocular opaque HMD (Oculus Rift) with a head tracking sensor. We modeled a tornado as the same 3DCG with the same 

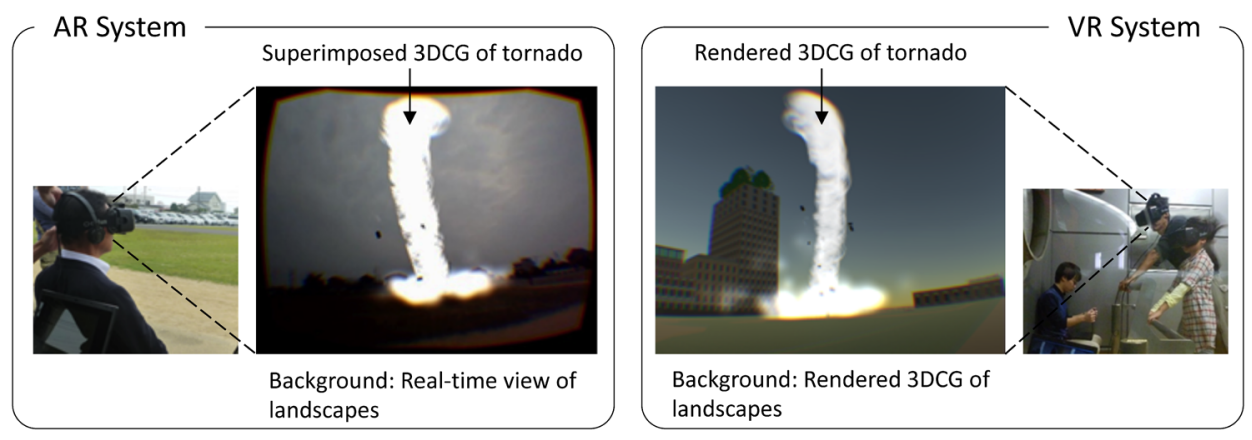

Figure 4. Screenshots of VR and AR Systems.

sound effects, and stereoscopically rendered it based on the following process:

1. A small cloud starts swirling with the sound of wind roaring above a learner.

2. The swirling cloud expands; raising debris and reaches the ground, indicating that a tornado has occurred.

3. For approximately one minute, the tornado gradually approaches but does not engulf the learner.

4. The tornado starts moving away from the learner while diminishing.

5. After approximately one minute, the tornado disappears.

Learners simply viewed the rendered tornado through the HMD without operating or interacting with the system in any way. Figure 4 shows screenshots of the VR and AR systems.

\subsubsection{VR System}

We modeled a virtual world in which a tornado occurred, imitating the facility's outside scenery (a large playground) since we used the AR system outside the facility building. Participants could see a 360-degree view of the virtual world by moving their heads.

\subsubsection{AR System}

We attached a stereoscopic camera to the HMD to capture the real-time view. When a participant wearing the HMD looked up at the sky, the AR system started superimposing the tornado to adjust the initial position of the superimposition and match the lowest part of the tornado to the ground. Participants could see a 360-degree view of the superimposed world by moving their heads.

\subsection{Settings}

We conducted the experiments as experience-based exhibitions at the facility. We ran two inside booths for C-STE and VR-STE and one outside booth for AR-STE. The C-STE booth was prepared in the wind-generation room.

\subsubsection{Procedure}

We explained the health risks of HMDs to the visitors to our booths, who ranged from children to the elderly. The experiment participants were 13 years and older. We performed the experiments taking the following steps that were common to all the booths:

1. Participants completed a pre-questionnaire asking about their tornado knowledge, experience, etc.

2. The participants received a short lecture on the mechanism by which tornadoes occur and the damage they have caused in the past.

3. The participants wore the HMD and looked into the HMD screen while moving their heads to adjust to the view.

4. The participants watched the rendered tornado, from occurrence to disappearance.

5. After taking off the HMD, the participants completed a post-questionnaire regarding their fear, self-efficacy, and learning motivation. Moreover, they were asked to subjectively describe their opinions on the STE. 
Table 1. Participant Demographics Obtained from Pre-questionnaire.

\begin{tabular}{lccccc}
\hline \multicolumn{1}{c}{ Group } & $\mathrm{N}$ & \multicolumn{1}{c}{ Age } & $\begin{array}{c}\text { Tornado } \\
\text { knowledge }\end{array}$ & $\begin{array}{c}\text { Tornado } \\
\text { experience }\end{array}$ & $\begin{array}{c}\text { Typhoon } \\
\text { experience }\end{array}$ \\
\hline C-STE (Control) & 13 & $M e d .=39(M . R .=28.5)$ & $Y e s=11, N o=2$ & Yes $=0, N o=13$ & $Y e s=11, N o=2$ \\
VR-STE (VR experimental) & 14 & $M e d .=23.5(M . R .=17.2)$ & $Y e s=13, N o=1$ & $Y e s=2, N o=12$ & $Y e s=11, N o=3$ \\
AR-STE (AR experimental) & 23 & $M e d .=35(M . R=28.7)$ & $Y e s=21, N o=2$ & $Y e s=1, N o=22$ & $Y e s=19, N o=4$ \\
\hline
\end{tabular}

Polar question about tornado knowledge: Do you know about tornadoes?

Polar question about tornado experience: Have you ever experienced (encountered) a tornado?

Polar question about typhoon experience: Have you ever experienced (encountered) a typhoon?

Med., median; M.R., mean rank

Table 2. Medians and Mean Ranks of Participants' Five-degree Replies in Pre- and Post-questionnaires.

\begin{tabular}{|c|c|c|c|c|c|c|}
\hline \multirow{2}{*}{ Group } & \multicolumn{2}{|c|}{ Fear } & \multicolumn{2}{|c|}{ Self-efficacy } & \multicolumn{2}{|c|}{ Learning motivation } \\
\hline & Pre-Q1 & Post-Q1 & Pre-Q2 & Post-Q2 & Pre-Q3 & Post-Q3 \\
\hline C-STE & $\begin{array}{l}\text { Med }=5 \\
(M . R .=31.0)\end{array}$ & $\begin{array}{l}\text { Med }=5 \\
(M . R .=28.7)\end{array}$ & $\begin{array}{l}\text { Med }=2 \\
(M . R .=27.9)\end{array}$ & $\begin{array}{l}\text { Med }=2 \\
(M \cdot R .=30.1)\end{array}$ & $\begin{array}{l}\text { Med. }=5 \\
(M . R .=33.2)\end{array}$ & $\begin{array}{l}\text { Med. }=5 \\
(M . R .=33.0)\end{array}$ \\
\hline VR-STE & $\begin{array}{l}\text { Med. }=5 \\
(M . R .=22.0)\end{array}$ & $\begin{array}{l}\text { Med. }=5 \\
(M \cdot R .=25.0)\end{array}$ & $\begin{array}{l}\text { Med }=2 \\
\text { (M.R. }=24.2)\end{array}$ & $\begin{array}{l}\text { Med. }=1.5 \\
(M . R .=19.7)\end{array}$ & $\begin{array}{l}\text { Med. }=4 \\
(M . R .=21.1)\end{array}$ & $\begin{array}{l}\text { Med. }=4.5 \\
(M . R .=24.8)\end{array}$ \\
\hline AR-STE & $\begin{array}{l}\text { Med }=5 \\
(M . R .=24.4)\end{array}$ & $\begin{array}{l}\text { Med }=5 \\
(M . R .=23.9)\end{array}$ & $\begin{array}{l}\text { Med }=2 \\
(M . R .=24.8)\end{array}$ & $\begin{array}{l}M e d .=2 \\
(M . R .=26.3)\end{array}$ & $\begin{array}{l}\text { Med. }=4 \\
(M . R .=23.7)\end{array}$ & $\begin{array}{l}\text { Med. }=4 \\
(M . R=21.5)\end{array}$ \\
\hline
\end{tabular}

Pre- and post-questions (Pre-Q1 and Post-Q1) about fear: Do you feel fear against tornado?

Pre- and post-questions (Pre-Q2 and Post-Q2) about self-efficacy: Can you protect yourself from a tornado?

Pre- and post-questions (Pre-Q3 and Post-Q3) about learning motivation: Do you want to learn tornado?

Options in the pre- and post-questions: $1=$ definitely no, $2=$ no, $3=$ neutral, $4=y e s, 5=$ definitely yes

Med., median; M.R., mean rank

Table 3. Decrement and Increment between Pre- and Post-questionnaires.

\begin{tabular}{|c|c|c|c|c|c|c|}
\hline \multirow{2}{*}{$\begin{array}{c}\text { Group } \\
\text { (no. of partici- } \\
\text { pants) }\end{array}$} & \multicolumn{2}{|c|}{ Fear } & \multicolumn{2}{|c|}{ Self-efficacy } & \multicolumn{2}{|c|}{ Learning motivation } \\
\hline & Decrement & Increment & Decrement & Increment & Decrement & Increment \\
\hline C-STE (13) & $-1(2,15.3 \%)$ & N/A & $\begin{array}{l}-2(2,15.3 \%) \\
-1(2,15.3 \%)\end{array}$ & $\begin{array}{l}+4(1,7.6 \%) \\
+3(1,7.6 \%) \\
+1(2,15.3 \%)\end{array}$ & N/A & $\begin{array}{l}+2(1,7.6 \%) \\
+1(1,7.6 \%)\end{array}$ \\
\hline VR-STE (14) & $-1(2,14.2 \%)$ & $+1(2,14.2 \%)$ & $\begin{array}{l}-2(1,7.1 \%) \\
-1(3,21.4 \%)\end{array}$ & $+1(1,7.1 \%)$ & $-3(1,7.1 \%)$ & $\begin{array}{l}+2(1,7.1 \%) \\
+1(6,42.8 \%)\end{array}$ \\
\hline AR-STE (23) & $-1(4,17.3 \%)$ & $+1(2,8.6 \%)$ & $\begin{array}{l}-2(1,4.3 \%) \\
-1(4,17.3 \%)\end{array}$ & $\begin{array}{l}+4(1,4.3 \%) \\
+2(2,8.6 \%) \\
+1(4,17.3 \%)\end{array}$ & $-1(2,8.6 \%)$ & $\begin{array}{l}+2(1,4.3 \%) \\
+1(6,26.0 \%)\end{array}$ \\
\hline
\end{tabular}

In main cells, decremental/incremental value (number of corresponding participants, rate truncated to the first decimal place)

\subsubsection{Participants}

Fifty visitors (13 to 79 years old) participated in the experiments. The numbers of participants in C-STE (the control group), VR-STE (the VR experimental group), and AR-STE (the AR experimental group) were 13,14 , and 23 , respectively. The uneven distribution of the participants were due to the booth locations. The
AR-STE booth was installed in a prominent location and noticed by more visitors.

Table 1 shows the participant demographics obtained from the pre-questionnaire. The medians of the participants' ages in C-STE, VR-STE, and AR-STE were $39,23.5$, and 35, respectively. A Steel-Dwass test (multiple comparisons) revealed no significant differ- 
ences; however, there were considerable differences, $p=0.064$ and $p=0.074$, comparing VR-STE with C-STE and AR-STE. For the polar questions, Fisher's exact test revealed no significant differences among the groups. Most participants had known about but not experienced a tornado; a few of the participants had tornado experience, but they may have been confused with dust devils. Several participants had experienced typhoons and seemed to know what tremendous winds would feel like. These results showed that participants were almost homogeneous from a demographic point of view.

\section{Results}

We analyzed the questionnaire results with nonparametric statistics by assuming a random population distribution and heteroscedasticity. Table 2 shows the medians and mean ranks of the participants' five-degree replies to the pre- and post-questionnaires.

The high medians of Pre-Q1 indicate that the participants have a considerable level of fear of tornadoes, and the low medians of Pre-Q2 suggest that the participants were unaware of what to do during a tornado. The high medians of Pre-Q3 may result from the participants' low self-efficacy and the fact that they had been motivated to learn disaster management.

\subsection{Inner-group Comparison}

We compared the medians of paired questions, such as Pre-Q1 and Post-Q1, to examine whether each STE influenced the survey variables. The medians of fear and learning motivation were found to remain high, whereas those of self-efficacy remained low. Furthermore, no remarkable differences were found in each group. For VR-STE, the medians of Post-Q2 and Post-Q3 slightly decreased and increased by 0.5 , respectively. For all the paired questions, a Wilcoxon signed-rank test revealed no significant differences.

\subsection{Intergroup Comparison}

We compared the medians of the paired questions among the groups in the post-questionnaire to examine which STE most influenced the survey variables. The medians of Post-Q1, Post-Q2, and Post-Q3 were 5, 1.52 , and 4-5, respectively. Based on the Steel-Dwass test, a significant difference was found between C-STE and
Table 4. Decrement in Self-efficacy (SE) and Change in Learning Motivation (LM).

\begin{tabular}{ccc}
\hline & $\begin{array}{c}\text { Decrement in SE } \\
\text { (Pre-Q2, Post-Q2) }\end{array}$ & $\begin{array}{c}\text { Change in LM } \\
\text { (Pre-Q3, Post-Q3) }\end{array}$ \\
\hline VR-STE & $-1(3,2)$ & $1(3,4)$ \\
& $-2(4,2)$ & $2(2,4)$ \\
AR-STE & $-1(2,1)$ & $1(4,5)$ \\
& $-1(2,1)$ & $1(4,5)$ \\
& $-1(4,3)$ & $0(4,4)$ \\
& $-1(2,1)$ & $1(3,4)$ \\
& $-2(3,1)$ & $1(3,4)$ \\
& $-1(4,3)$ & $1(3,4)$ \\
\end{tabular}

AR-STE $(p<0.05)$ in the learning motivation.

Table 3 shows the changes (decrement and increment) in the participants' replies between the pre- and post-questionnaires. Concerning fear, all the groups were found to have decreased by 1 , and the highest rate of decrease was found in AR-STE $(17.3 \%)$. Regarding self-efficacy, the changes ranged from -2 to +4 . The highest rates of decrement and increment were found in VR-STE (21.4\%) and AR-STE (17.3\%), respectively. For C-STE, VR-STE, and AR-STE, the total rates of decrement and increment were $30.7 \%$ and $30.7 \%$, $28.5 \%$ and $7.1 \%$, and $21.7 \%$ and $30.4 \%$, respectively. Finally, concerning learning motivation, the changes ranged from -3 to +2 and were observed to have mainly increased. The highest rate of increment was observed in VR-STE (42.8\%).

\subsection{Participants' Opinions}

Eight, eleven, and two participants gave their opinions in the C-STE, VR-STE, and AR-STE groups, respectively. We assumed that the low number in the AR-STE group was because the participants hesitated to spend more time for the description at the outdoor booth due to the prevailing inclement weather conditions. Dominant opinions in each group were as follows:

(1) C-STE

- I felt fear of the tornado's wind.

- The real wind made the experience realistic.

- We should treat tornado alerts more seriously.

- We should learn how to protect our lives from tornadoes.

(2) VR-STE

- I felt fear of flying debris caused by tornadoes. 
- I felt fear of the tornado's unpredictable movement.

- The tornado was more tremendous than I thought.

- I now know that tornadoes are so scary.

(3) AR-STE

- The tornado was more tremendous than I thought.

\section{Considerations}

We answer each RQ by considering the questionnaire results.

\subsection{Audiovisual Effects and Fear (RQ1)}

In response to RQ1, we aimed to clarify the relationship between audiovisual effects and fear. In the inner-group and intergroup comparisons, the medians of Pre-Q1 and Post-Q1 were high and showed no significant differences. This shows that VR- and AR-STEs in the median are equal to C-STE - which has shown the highest level of fear - and have maintained the participants' fear with audiovisual effects without real wind. One may conclude that the audiovisual effects expressed the tornado realistically. In each group, however, two to four participants felt less fear. This indicates that the STEs do not necessarily express a tornado beyond the learners' imagination, i.e., their current fear. In other words, without accessing their current internal state, the STEs can hardly provide a moderate level of fear. Meanwhile, since the medians of Post-Q2 are not high, we should emphasize that the STEs do not make learners feel optimistic about tornadoes. Fear provided by the STEs may be sufficient for self-efficacy and learning motivation.

Now, we answer RQ1 as follows: audiovisual effects can, but do not always, maintain learners' fear of tornadoes.

\subsection{Fear and Transition to Learning (RQ2)}

With RQ2, we aimed to clarify the relationship between fear and the transition to learning while considering the answer to RQ1 provided above.

Concerning self-efficacy, the medians of Pre-Q2 and Post-Q2 were low and showed no significant differences in the inner-group and intergroup comparisons. This shows that in the median, VR- and AR-STEs are equal to C-STE and maintained the participants' selfefficacy by their fear. However, in each group, some participants felt their self-efficacy was lowered or heightened. In C-STE and AR-STE, decrement and increment tended to occur evenly, whereas in VR-STE, decrement was dominant. This indicates that fear can influence self-efficacy but lacks coherence. Supposing that self-efficacy is inversely influenced by fear, we calculated the correlation coefficients between the replies in Post-Q1 and Post-Q2. The correlation coefficients were obtained as $0.39(p=0.18), 0.007 \quad(p=0.97)$, and $-0.19(p=0.38)$ in C-STE, VR-STE, and AR-STE, respectively. Furthermore, the participants of C-STE had a weak positive correlation, which indicates that fear can arouse self-efficacy. However, this indication does not appear to apply to VR- and AR-STEs and, thus, differs from our supposition. Considering the p-value (test for no correlation) of C-STE, we cannot always approve of the obtained correlation. It should be noted that we have not yet found the cause and will have to conduct further analysis and/or experiments to that end.

Concerning learning motivation, the medians of Pre-Q3 and Post-Q3 were high and showed no significant differences except for between C-STE and AR-STE. This indicates that VR-STE is almost equivalent to C-STE and maintained the participants' learning motivation by their fear. AR-STE also retained the participants' learning motivation; however, it is not equivalent to C-STE. For each of the VR- and AR-STEs, seven participants experienced heightened learning motivation. Table 4 shows the changes in the learning motivation of the participants of the VR- and AR-STEs who experienced lowered self-efficacy. Four participants of C-STE who experienced lowered self-efficacy had no changes; their values of Pre-Q3 and Post-Q3 remained 5. Meanwhile, all four participants in VR-STE and the three participants in AR-STE experienced heightened learning motivation. This shows that, for VR- and AR-STEs, lowered self-efficacy can cause heightened learning motivation. One may think for C-STE, no changes were due to a ceiling effect.

Hence, we answer RQ2 as follows: fear of tornadoes can influence learners' self-efficacy and learning motivation; however, the influence is yet to be clarified.

\subsection{More Suitable STE (RQ3)}

The questionnaire results indicate that VR- and AR-STEs are equivalent to C-STE. In other words, audiovisual effects can realistically express tornadoes 
accompanied by tremendous wind and still retain learners' fear. Therefore, the C-STE, which is available only in a wind-generation room, can be replaced with either VR-STE or AR-STE which are available at various locations. We propose that ease of access, such as a system's in-home availability, is crucial for STE popularization.

The participants' opinions revealed what aroused their fear: for participants of C-STE, who were exposed to the real wind, it was the tornado's wind; while for the participants of VR- and/or AR-STEs, it was the tornado's visual aspects, i.e., flying debris, movement, and size. We found the common dominant opinion (unthought tremendousness of tornado) in VR- and AR-STEs, but not in all the groups. Dominant opinions in C-STE mentioned what they should do. This tendency shows that regarding the presence or absence of real wind, C-STE can provide experiences different from VR- and AR-STEs and furthermore C-STE makes it easier for learners to shift to Step 2 than VR- and AR-STEs. Considering their opinions, we think that VR- and AR-STEs enable learners to feel fear and observe a tornado under safe and stable conditions.

To enable uniform experimental conditions in the three STEs, the modeled tornado did not destroy anything around the playground. Unlike AR-STE, C-STE and VR-STE could express such destruction. If C-STE and VR-STE expressed such destruction, the participants could have felt more fear. This shows that C-STE and VR-STE have more factors to control fear than AR-STE.

Furthermore, there were no significant differences between VR- and AR-STEs. In terms of system operation, we opine that VR-STE outperforms AR-STE, which is frequently conducted outside and is easily affected by weather conditions. In addition, the current AR-STE system requires learners to adjust their view to ensure high geometric consistency, i.e., to express a tornado with high visual reality.

Therefore, we answer RQ3 as follows: VR-STE is more suitable than AR-STE in terms of fear controllability and system operation.

\subsection{Limitations}

Visitors to the facility arbitrarily participated in the experiments, i.e., in one of the three booths. Thus, the participants were not enough in number and unregu- lated. In particular, the participants were not homogenized in age. Meanwhile, when they visited the facility to practice disaster management, the participants were considerably homogenized in fear, self-efficacy, and learning motivation. For fear, this situation caused a ceiling effect. With a broader sample of participants, i.e., naturally distributed, we could have performed the experiments in terms of their fear, anxiety, and other internal states. Additionally, the questionnaire had to be minimal and simple to ensure that the participants have enough time to look around the facility. Therefore, we did not survey and analyze fear, self-efficacy, and learning motivation in detail and also the effectiveness of the questionnaire survey (self-reporting method) for surveying the participants' internal state. Chittaro and Buttussi adopted biological sensors such as electrodermal activity to obtain physiological arousal data from learners ${ }^{(14)}$. Nevertheless, we maintain that, to interpret the experimental results more objectively, other kinds of data of learners will need to be required.

\section{Conclusion}

We presented the experimental results on RQs that aimed to clarify how VR- and AR-STEs (audiovisual effects) differ from C-STE (audiovisual effects with real wind) in terms of fear, self-efficacy, and learning motivation. The experimental results, i.e., our answers, are summarized as follows: Audiovisual effects retain learners' fear of a tornado and can influence learners' selfefficacy and learning motivation. Furthermore, we confirm that VR-STE is more suitable than AR-STE.

VR-STE, AR-STE, and C-STE can inspire learners to take Step 2, i.e., turn their thoughts to evacuation. However, seeing a tornado through VR or AR is as insufficient as ICTDE. The comparative experiments of the STEs were focused on Step 1, which should lead to Steps 2 and 3, i.e., enable learners to think how to evacuate and then actually evacuate in the virtual or real world while providing a moderate level of fear. We are still in an early stage and must continue to the next stage in which STE leads to learning how to evacuate from natural disaster sites. The experimental results showed that VR- and AR-STEs do not increase the participants' self-efficacy. To establish STE as practicable disaster education, the model (relationships) among fear, selfefficacy, and learning motivation have to be clarified. Additionally, learning supports that enable learners to 


\section{COMPARATIVE EXPERIMENTS ON SIMULATED TORNADO EXPERIENCE VIA VIRTUAL REALITY AND AUGMENTED REALITY}

experience unshaken self-efficacy as learning outcome need to be developed. Although we did not sufficiently discuss how STE provides a moderate level of fear, we showed that people who experience not-too-little fear of tornadoes could lower their self-efficacy, but heighten their learning motivation. However, those who do not feel fear can also lower their learning motivation due to their high self-efficacy and, in some cases, such people, especially, must learn how to evacuate. The relationships among the three variables - fear, self-efficacy, and learning motivation - are not simple. To determine the optimum level, the relationships should be represented as a feasible model, and then larger-scaled experiments should be conducted with more diverse participants.

In recent years, immersive devices for $\mathrm{VR}$ and $\mathrm{AR}$, such as smartphone-based HMDs and smart glasses, have been produced and rapidly popularized. In future research, we plan to improve on the VR and AR systems used in the experiments by introducing such immersive devices. We strongly anticipate the wide acceptance of the improved VR- and AR-STEs by the public in the near future, as it will contribute immensely to their ability to survive tornadoes.

\section{Acknowledgment}

This study was supported in part by the Grant-in-Aid for Scientific Research (B) No. 18H01054 from the Japan Society for the Promotion of Science. We express our thanks to Tokushima Prefectural Disaster Prevention Center and S. Iwama for their cooperation in this study.

\section{References}

(1) Munich, R. E.: Topics Geo: Natural Catastrophes 2017, München, Germany (2017).

(2) Japan Meteorological Agency (JMA): "Seismicity in Japan in 2018", https:/www.jma.go.jp/jma/press/1901/11a/ 1812jishin2018.pdf (accessed 2020.2.17). (in Japanese)

(3) Mas, E., Adriano, B. and Koshimura, S.: "An Integrated Simulation of Tsunami Hazard and Human Evacuation in La Punta, Peru", J. of Disaster Research, Vol. 8, No. 2, pp. 285-295 (2013).

(4) Wahyudin, D. and Hasegawa, S.: "Mobile Serious Game Design for Training Ethical Decision Making Skills of Inexperienced Disaster Volunteers", J. of Information and Systems in Education, Vol. 14, No. 1, pp. 285-295
(2015)

(5) Okazaki, Y., Kozaki, S., Matsuo, S. et al.: "Prototyping of Community-based Hazard Mapping Support System for Traditional Towns with Local Heritage", Workshop Proc. of ICCE2017, pp. 171-179 (2017).

(6) Feng Z., Gonzáleza, V. A., Amorb, R. et al.: "Immersive Virtual Reality Serious Games for Evacuation Training and Research: A Systematic Literature Review", Computers \& Education, Vol. 127, pp. 252-266 (2018).

(7) Lovreglio, R.: "A Review of Augmented Reality Applications for Building Evacuation", Proc. of 17th Int. Conf. on Computing in Civil and Building Engineering (2018).

(8) JMA: "Number of Occurrence of Tornado in Japan in Each Year", http://www.data.jma.go.jp/obd/stats/data/ bosai/tornado/stats/annually.html (accessed 2020.2.17). (in Japanese)

(9) JMA: "Remarkable Examples of Tornado and Windflaw in Japan", http://www.data.jma.go.jp/obd/stats/data/bosai/ tornado/special/special_history.html (accessed 2020.2.17).

(10) Rüstemli, A. and Karanci, N. A.: "Correlates of Earthquake Cognitions and Preparedness Behavior in a Victimized Population", J. of Social Psychology, Vol. 139, No. 1, pp. 91-101 (1999).

(11) Johnson, V. A., Ronan, K. R., Johnstion, D. M. et al.: "Evaluations of Disaster Education Programs for Children: A Methodological Review", Int'1. J. of Disaster Risk Reduction, Vol. 9, pp. 107-123 (2014).

(12) Cvetković, V. M., Öcal, A. and Ivanov, A.: "Young Adults' Fear of Disasters: A Case Study of Residents from Turkey, Serbia and Macedonia", Int'1. J. of Disaster Risk Reduction, Vol. 35, Article 101095 (2019).

(13) Chittaro, L. and Sioni, R.: "Serious Games for Emergency Preparedness: Evaluation of an Interactive vs. a Noninteractive Simulation of a Terror Attack", Computers in Human Behavior, Vol. 50, pp. 508-519 (2015).

(14) Chittaro, L. and Buttussi, F.: "Assessing Knowledge Retention of an Immersive Serious Game vs. a Traditional Education Method in Aviation Safety", IEEE Transactions on Visualization and Computer Graphics, Vol. 21, No. 4, pp. 529-538 (2015).

(15) Hirokane, M., Nishiwaki, K. and Endo, T.: "Experiment of Disaster Prevention Education and Evaluation Using a Torrential Rain Simulated Experience Application", Proc. of SCIS\&ISIS2016, pp. 432-437 (2015).

(16) Tornado VR: “Tornado VR-Mark McCallum”, http:// mccallummark.com/tornado-vr (accessed 2020.2.17).

(17) Taylor-Nelms, L. and Hill, V.: "Assessing 3D Virtual 
World Disaster Training Through Adult Learning Theory", Int'1. J. of Serious Games, Vol. 1, No. 4, pp. 3-16 (2014).

(18) Dong, H., Schafer, J. and Ganz, A.: “Augmented Reality based Mass Casualty Incident Training System", Proc. of 2016 IEEE Symposium on Technologies for Homeland Security, pp. 1-4 (2016).

(19) Milgram, P. and Kishino, F.: "A Taxonomy of Mixed Reality Virtual Displays", IEICE Transactions on Information and System, Vol. E77-D, No. 12, pp. 13211329 (1994).

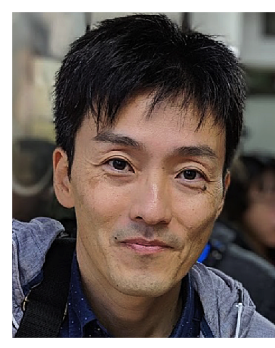

Hiroyuki Mitsuhara received his B.E. and M.E. degrees from Kindai University, Japan in 1998 and 2000, respectively, followed by the Ph.D. degree from Tokushima University, Japan, in 2003. He was a visiting academic at Curtin University, Australia, in 2017-2018. He is currently an associate professor at Tokushima University. His research interests include VR/AR for education, game-based learning, human-computer interaction, and disaster education.

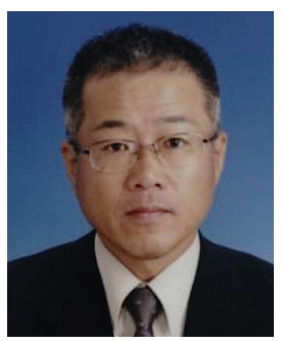

Masami Shishibori was born in 1965. He received his BS Degree in 1991, his MS Degree in 1993, and $\mathrm{PhD}$ Degree in 1997 from Tokushima University, Japan. He is currently a Professor in Graduate School of Technology, Industrial and Social Sciences, Tokushima University, Japan. His research interests include multimedia processing, information retrieval, and natural language processing. 